\title{
Frustration between syn- and anticlinicity in mixtures of chiral and non-chiral tilted smectic-C-type liquid crystals
}

\author{
Jan P. F. Lagerwall ${ }^{1}$, Gerd Heppke ${ }^{2}$, and Frank Giesselmann ${ }^{1}$ \\ 1 Institute of Physical Chemistry, University of Stuttgart, Pfaffenwaldring 55, D-70569 Stuttgart, Germany. \\ 2 Department of Chemistry, Technical University Berlin, Str. des 17. Juni 112, Berlin, Germany.
}

the date of receipt and acceptance should be inserted later

\begin{abstract}
We study the effects of mixing ferroelectric and antiferroelectric liquid crystal compounds (FLCs and AFLCs) when the former are strictly synclinic and the latter strictly anticlinic, i.e. one mixture component exhibits only $\mathrm{SmC}^{*}$ and the other only $\mathrm{SmCa}^{*}$ as tilted phase. Three different paths between syn- and anticlinicity were detected: transition directly between $\mathrm{SmC}^{*}$ and $\mathrm{Sm}-C_{\mathrm{a}}{ }^{*}$, transition via the $\mathrm{SmC}_{\beta} *$ and $\mathrm{SmC}_{\gamma}{ }^{*}$ subphases, or by 'escaping' the clinicity frustration by reducing the tilt to zero, i.e. the $\mathrm{SmA}^{*}$ phase is extended downwards in temperature, separating $\mathrm{SmC}^{*}$ from $\mathrm{SmCa}^{*}$ in the phase diagram. The most common path is the one via the subphases, demonstrating that these phases appear as a result of frustration between syn- and anticlinic and, consequently, between syn- and antipolar order. For assessing the role of chirality, we also replaced the FLC with non-chiral synclinics. With one of the AFLCs, the route via supbhases was detected even in this case, suggesting that chirality - although necessary - does not have quite the importance that has previously been attributed to the appearance of the subphases. The path chosen in the mixture study seemed to be determined mainly by the synclinic component, the subphase induction occurring only when the Sm- $A^{*-S m}-C^{*}$ transition was second-order.
\end{abstract}

PACS. 61.30.-v Liquids crystals - 77.80.-e Ferroelectricity and antiferroelectricity - 64.60.-i General studies of phase transitions

\section{Introduction}

The smectic $\mathrm{C}(\mathrm{SmC})$ liquid crystal phase, characterized by a non-zero tilt angle $\theta$ between the director $\mathbf{n}$ and the smectic layer normal $\mathbf{k}$, is today known to exist in several variants. The two most important are the synclinic $\mathrm{SmC}$ and the anticlinic $\mathrm{SmC}_{\mathrm{a}}$, with parallel and antiparallel tilt directions in adjacent layers, respectively. If these phases are made chiral $\left(\mathrm{SmC}^{*}\right.$ and $\left.\mathrm{SmC}_{\mathrm{a}}{ }^{*}\right)$ the director tilting direction precesses in a helical fashion along the smectic layer normal, with a pitch typically in the micrometer range. Moreover, each smectic layer is then spontaneously polarized with an electric polarization $\mathbf{P}_{s}$ $\propto \mathbf{n} \times \mathbf{k}$, and the chiral phases can thus be referred to also as syn- and antipolar, respectively ${ }^{1}$. By forcing the phases into a non-helical state by means of closely spaced substrates (surface-stabilization) the $\mathrm{SmC}^{*}$ and $\mathrm{SmC}_{\mathrm{a}}{ }^{*}$ phases can show ferroelectric and antiferroelectric behavior, respectively [1]. Compounds exhibiting only $\mathrm{SmC}^{*}$ are

1 In using this terminology, we refer to the structure when the helical modulation, another effect of chirality, is neglected. In real bulk samples the polarization directions of adjacent layers are not exactly parallel in $\mathrm{SmC}^{*}$, nor exactly antiparallel in $\mathrm{SmCa}^{*}$, due to the slow precession in the helical superstructure. therefore often referred to as ferroelectric liquid crystals (FLCs) whereas the term antiferroelectric liquid crystal (AFLC) is generally used for compounds exhibiting the $\mathrm{SmC}_{\mathrm{a}}{ }^{*}$ phase, although they often exhibit also other chiral smectic-C-type phases, including $\mathrm{SmC}^{*}$. It is still a matter of discussion what the origin and nature of the director tilt is [2] as well as which the relevant types of correlation in tilting directions across smectic layer boundaries are $[3-5]$.

From single-component AFLCs which exhibit both $\mathrm{SmC}^{*}$ and $\mathrm{SmC}_{\mathrm{a}}{ }^{*}$ phases we know of two paths between syn- and anticlinicity: either a direct transition or via the so-called 'subphases' $\mathrm{SmC}_{\beta} *$ and $\mathrm{SmC}_{\gamma} *$ (sometimes only one of them). ${ }^{2}$ The latter path is common in 'standard' alkyl chain AFLCs, but almost never observed in AFLCs with one or two fluorinated terminal chains $[8,9]$. The location of $\mathrm{SmC}_{\beta} *$ and $\mathrm{SmC}_{\gamma} *$ between $\mathrm{SmC}^{*}$ and $\mathrm{SmC}_{\mathrm{a}} *$ early on led to the natural suggestion that these phases appear as a result of competition / frustration between syn- and anticlinicity, or between syn- and antipolarity [10-12]. A third subphase, $\mathrm{SmC}_{\alpha}{ }^{*}$, may sometimes take the place of

\footnotetext{
${ }^{2}$ Many different nomenclatures exist for the AFLC phases. We find the original naming scheme proposed by the Fukuda school [6] to be the most logical and confusion-free, cf.the discussion in reference [7], and therefore use that in this paper.
} 


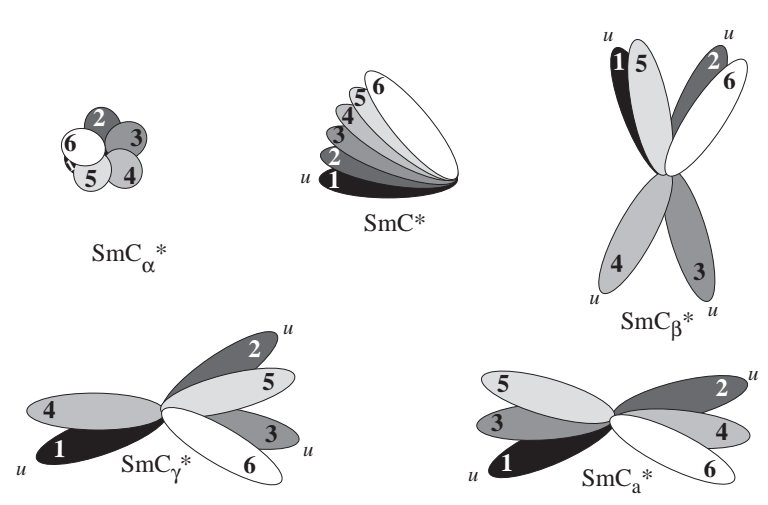

Fig. 1. Schematic overview of the director organizations in the five well-established chiral smectic-C-type phases (as they are understood today). The director tilting directions in six adjacent layers are illustrated with ellipses, the grey-shade of which illustrates the level of the layer (the layer normal is the paper plane normal): the back layer (1) is black and the front layer (6) is white. The layers constituting the smallest repeating unit (disregarding the helical modulation which introduces a constant distortion between and within the repeating units) are indicated with a $u$ at the end of each ellipse. Since the tilt direction modulation in $\mathrm{SmC}_{\alpha} *$ is incommensurate with the layer spacing it is difficult to define a repeating unit for this phase.

$\mathrm{SmC}^{*}$ as the first tilted phase forming on cooling from the non-tilted $\mathrm{SmA}^{*}$ phase. On further cooling, $\mathrm{SmC}_{\alpha} *$ (which essentially is a low-tilt and ultrashort pitch version of $\mathrm{SmC}^{*}$ ) is then followed by either $\mathrm{SmC}^{*}, \mathrm{SmC}_{\beta}$ * or $\mathrm{SmC}_{\mathrm{a}}{ }^{*}$. All three subphases usually have small temperature ranges, typically in the range $0.5-5 \mathrm{~K}$.

Thanks to sophisticated experimental investigations [13-15] one now has a relatively good idea of the complex director organization in the five chiral smectic-C-type phases (cf. Figure 1 where the present understanding is schematically summarized) but their theoretical descriptions are still under development. A few promising models have recently been designed, showing some overlap but also important differences $[3-5,16,17]$. Much empirical knowledge exists on how easily the subphases are destabilized and this has led to some conjectures regarding what factors are important for the formation of these phases, e.g. high enantiomeric excess [16] and high translational (smectic) order [7, 18].

In order to improve our understanding of the delicate balance between syn- and anticlinicity, including the nature and origin of the three subphases as well as the variety of phase sequences possible in AFLCs, we have performed a series of systematic mixing experiments where we let smectics with no smectic-C-type phase other than $\mathrm{SmC}_{\mathrm{a}}$ * meet compounds where the only tilted phase is $\mathrm{SmC}$ or SmC*. Some studies in this direction have been carried out earlier [10,19-23] but as the number of compounds was generally restricted to two (in one study, a total of three compounds were investigated) the conclusions are not of a very general nature. Moreover, in all previous studies but one, at least one of the components already on its own exhibited more than one type of tilted phase, generally $\mathrm{SmC}^{*}$ as well as $\mathrm{SmC}_{\mathrm{a}}{ }^{*}$, rendering the results less conclusive than in the present study. Here we present the investigation of all combinations of nine strictly synclinic compounds - chiral as well as non-chiral - with five strict anticlinics, giving a better overview of how the induced frustration can be resolved.

We find three possible paths between syn- and anticlinic order. In addition to the two usual ways, via the $\mathrm{SmC}_{\beta} *$ and $\mathrm{SmC}_{\gamma} *$ subphases or with a direct transition, we encountered a third path: several systems solved the clinicity frustration essentially by reducing the tilt to zero, i.e. the $\mathrm{SmA}^{*}$ phase was extended downwards in temperature at intermediate mixture ratios, separating $\mathrm{SmC}^{*}$ from $\mathrm{SmC}_{\mathrm{a}}{ }^{*}$ in the phase diagram. The most common route is however the one via $\mathrm{SmC}_{\beta}{ }^{*}$ and $\mathrm{SmC}_{\gamma}{ }^{*}$, supporting the idea that the fundamental requirement for observing these phases is the frustration between syn- and anticlinic order. Considering that AFLCs often have relatively high spontaneous polarization $P_{s}$, a somewhat surprising result is that $P_{s}$ has little importance for the path followed; one subphase-containing mixture had a saturation value of only $P_{s} \approx 10 \mathrm{nCcm}^{-2}$. As interesting side-effect the mixture experiments have given us a means of extending the temperature range of the subphases considerably up to about $50 \mathrm{~K}$.

\section{Experimental}

Dielectric spectroscopy with simultaneous texture monitoring was carried out using an HP 4192A impedance bridge and FLC Electronics DiScO measurement software. The optical tilt angle $\theta$ and the spontaneous polarization $P_{s}$ were measured using methods described in references [24] and [25], respectively. Small-angle x-ray scattering (SAXS) experiments were carried out on samples filled into Mark capillary tubes with $0.7 \mathrm{~mm}$ diameter, using $\mathrm{Cu}-K_{\alpha}$ radiation, a Kratky compact camera and an M. Braun electronic 1D detector.

The selected compounds and mixtures were filled into polyimide-coated (for planar alignment) electrode-equipped cells (Chalmers MC2 assembly line) for texture observations, dielectric spectroscopy and measurements of the order parameters $\theta$ and $P_{s}$. For dielectric spectroscopy the thickness was $23.5 \mu \mathrm{m}$, otherwise it was $2.5 \mu \mathrm{m}$ or $4 \mu \mathrm{m}$. The sample temperature was regulated with Instec, Linkam or Julabo hot stages, mounted on an Olympus BH-2 polarizing microscope. In order to get estimates of the mesogen lengths, the conformations of the different molecular structures were optimized with MOPAC / AM1.

\section{Selected compounds}

The compounds under investigation are listed in Table 1 (anticlinics) and Table 2 (synclinics). In order to keep the study in reasonable proportions we limited the selection 
Table 1. Anticlinic compounds used in the study. Transition temperatures in ${ }^{\circ} \mathrm{C}$, polarization $P_{s}$ in $\mathrm{nC} \mathrm{cm}^{-2}$ and tilt angle $\theta$ in degrees (for the two latter parameters their highest values are listed; $\mathrm{nm}=$ not measured). The last column lists the order of the transition to the $\mathrm{SmCa} / \mathrm{SmCa}^{*}$ phase.

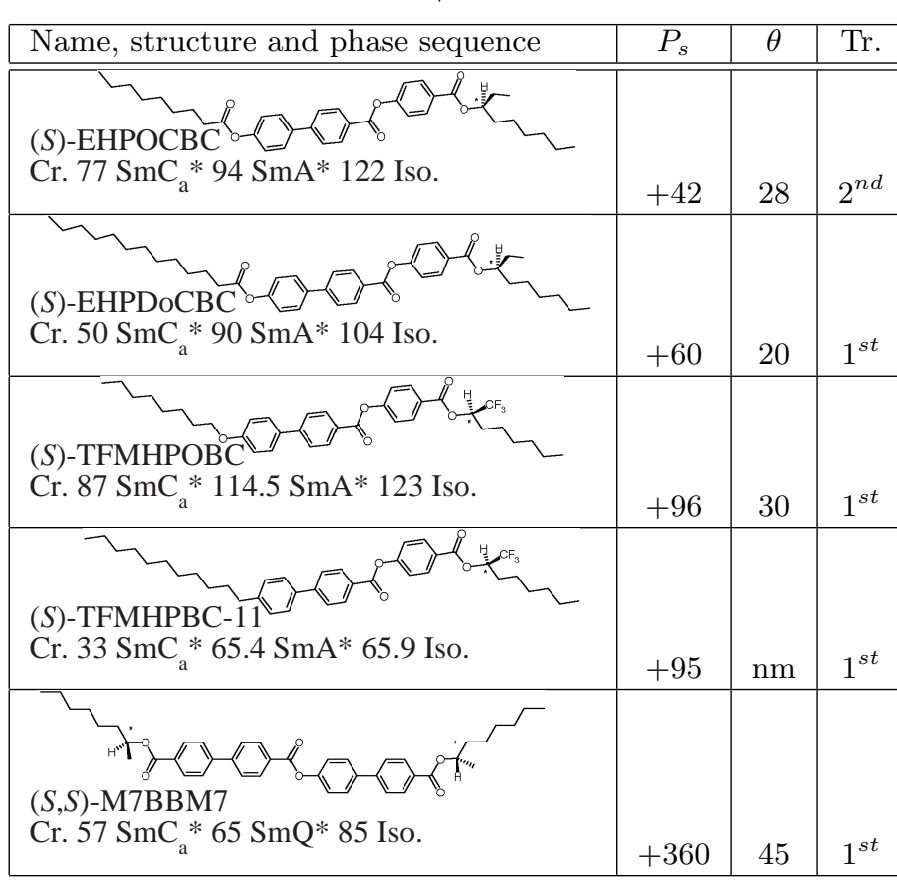

of chiral compounds to $(S)$ enantiomers. Exchanging one of the components with the other enantiomer will be an interesting and most relevant follow-up study. As anticlinics we chose four compounds which are structurally similar to the well-known AFLC compound MHPOBC, but which all exhibit direct $\mathrm{SmA}^{*}-\mathrm{SmC}_{\mathrm{a}}{ }^{*}$ transitions, as well as a bistereogenic compound where the chiral methylheptyloxycarbonyl tail of MHPOBC is attached on both sides of a core consisting of two esterlinked biphenyls. This compound has very large $P_{s}$ and $\theta$ and a SmQ*$\mathrm{SmC}_{\mathrm{a}} *$ phase sequence (no SmA* phase). Unfortunately, the transition to $\mathrm{SmC}_{\mathrm{a}}{ }^{*}$ is kinetically inhibited making direct transitions between crystal and $\mathrm{SmQ}^{*}$ common, thereby rendering investigations of the $\mathrm{SmC}_{\mathrm{a}}{ }^{*}$ phase in the pure compound very difficult.

For the synclinic materials larger variations in structure were possible, since compounds with $\mathrm{SmC} / \mathrm{SmC}^{*}$ as only tilted smectic phase are ubiquitous. The first molecule in Table 2 is the 'classic' FLC compound DOBAMBC. Next, three compounds were chosen from a series of FLCs with chlorocarbon acid ester chiral tails, all exhibiting relatively high spontaneous polarization but varying quite dramatically in the phase sequence. Two FLCs with cores identical to MHPOBC were investigated, both having much longer achiral chain than MHPOBC, attached to the core via an ester linkage. The long achiral chain is enough to destabilize all tilted phases other than $\mathrm{SmC}^{*}$ in MHPOd$\mathrm{CBC}$, and in IPC-16 the chiral tail has in addition been replaced with a camphor group. W504 is a somewhat special FLC, with fluorine substituents in the core as well as
Table 2. Synclinic compounds used in the study. Transition temperatures in ${ }^{\circ} \mathrm{C}$, polarization $P_{s}$ in $\mathrm{nC} \mathrm{cm}^{-2}$ and tilt angle $\theta$ in degrees (for the two latter parameters their highest values are listed; $\mathrm{nm}=$ not measured). $\mathrm{SmX}$ is used as a generic label for unidentified higher-ordered smectic phases. The last column lists the order of the transition to the $\mathrm{SmC} / \mathrm{SmC}^{*}$ phase.

\begin{tabular}{|c|c|c|c|}
\hline Name, structure and phase sequence & $P_{s}$ & $\theta$ & Tr. \\
\hline $\begin{array}{l}\text { (S)-DOBAMBC } \\
\text { Cr. } 76\left(\mathrm{SmI}^{*} 63\right) \mathrm{SmC}^{*} 95 \mathrm{SmA}^{*} 117 \text { Iso. }\end{array}$ & -7 & 28 & $2^{n d}$ \\
\hline Cr. 76 (SmG* $\left.65 \mathrm{SmC}^{*} 70\right) \mathrm{SmA} * 82$ Iso. & -150 & 20 & $1^{s t}$ \\
\hline $\begin{array}{l}\text { Cr. } 96 \text { (SmF* } 69 \text { SmI* 74) SmC* } \\
147 \mathrm{~N}^{*} 183 \mathrm{BP}^{*} 185 \text { Iso. }\end{array}$ & -250 & $\mathrm{~nm}$ & $1^{s t}$ \\
\hline $\begin{array}{l}\text { Cr. } 62 \text { SmX* } 75 \text { SmC* } 119 \text { SmA* }^{*} \\
131 \mathrm{~N}^{*} 136 \text { Iso. }\end{array}$ & -160 & 34 & $1^{\text {st }}$ \\
\hline Cr. $100 \mathrm{SmC} * 140.5 \mathrm{SmA}^{*} 168$ Iso. & -50 & 30 & $2^{n d}$ \\
\hline $\begin{array}{l}(S) \text {-MHPOdCBC } \\
\text { Cr. } 68 \text { (SmI* 66.5) SmC* } \\
114 \text { SmA* } 124 \text { Iso. }^{*}\end{array}$ & +95 & 32 & $2^{n d}$ \\
\hline $\begin{array}{l}(S)-\mathrm{W} 504 \\
\text { Cr. } 103 \mathrm{SmC}^{*} 121.5 \mathrm{SmA}^{*} 146 \text { Iso. }\end{array}$ & -200 & 28 & $2^{n d}$ \\
\hline $\begin{array}{l}8 \mathrm{OPhPy} 10 \\
\mathrm{Cr} .37 \mathrm{SmC} 68.5 \mathrm{SmA} 73.5 \text { Iso. }\end{array}$ & 0 & $\mathrm{~nm}$ & $2^{n d}$ \\
\hline $\begin{array}{l}\text { Cr. } 74 \text { SmC } 95 \text { N } 124 \text { Iso. } \\
\text { COAB }\end{array}$ & 0 & 45 & $1^{s t}$ \\
\hline
\end{tabular}

in the achiral tail. This compound, which we describe in detail in a separate paper [26], exhibits a tilting transition that follows the asymmetric diffuse cone (ADC) model of Adriaan de Vries [27-29], characterized by small change in actual molecule tilt, the non-zero director tilt instead being a result of azimuthal biasing of tilting directions. Finally, two achiral synclinics were added to the set, one with SmA-SmC and one with N-SmC phase sequence. The former exhibits a second-order tilting transition giving a moderate saturation value of $\theta$, whereas the latter compound exhibits an essentially constant tilt of about $45^{\circ}$ in the $\mathrm{SmC}$ phase. 


\section{Results and discussion}

\subsection{Mixtures of syn- and anticlinics}

\subsubsection{Texture investigations in contact samples}

The main basis for determining the transition path for each possible combination of the nine synclinics and the five anticlinics was texture studies in homeotropic contact samples, cf. the examples in Figure 2. When the clinicity change took place via the subphases these phases were recognized in the contact samples through their characteristic long-pitch grey-white schlieren textures. They generally constituted a striking contrast to the adjacent $\mathrm{SmC}^{*}$ and $\mathrm{SmC}_{\mathrm{a}}{ }^{*}$ textures, which in these mixtures often had short pitch helices. In many cases the pitch even decreased on approaching the phase transition, as in the example of $(S)$-TFMHPOBC mixed with $(S)$-DOBAMBC in Figure 2a. The detection of the boundary between $\mathrm{SmC}_{\beta}$ * and $\mathrm{SmC}_{\gamma} *$ was in some systems much more difficult. The textures of these two phases can be very similar and the width of each of the phases in the contact sample could vary considerably, such that the texture sometimes suggested the existence of only one of the subphases. In order to establish the exact nature of the phase or phases between $\mathrm{SmC}^{*}$ and $\mathrm{SmC}_{\mathrm{a}}{ }^{*}$, we therefore prepared contact samples between a selected number of subphase-producing mixtures with defined composition and well-characterized reference AFLCs. This allowed us to verify by means of miscibility tests that indeed both subphases were generated in all combinations tested in this way. Moreover, we ran dielectric spectroscopy runs on the selected mixtures and this generally also gave a good verification of the subphase nature, as described below.

At temperatures of the tilted phases, contact samples with the combinations leading to a direct clinicity change - the least common route - typically exhibited homeotropic textures with two broad areas separated by a sharp line indicating the $\mathrm{SmC}^{*}-\mathrm{SmC}_{\mathrm{a}}$ * transition, cf. Figure 2c. The mixtures with extended $\mathrm{SmA}^{*}$ phase, finally, at first seemed to exhibit no other liquid crystalline phase down to crystallization. The homeotropic texture was constantly black and featureless in the center region of the contact sample and no transitional texture changes could be observed as the sample was cooled down. However, the more careful study on mixtures with well-defined composition (cf. Section 4.1.2.) revealed that other liquid crystalline phases, probably higher-ordered ones, formed at least in one of the systems. The combination of the bistereogenic anticlinic compound $(S, S)$-M7BBM7 with the achiral synclinic $8 \mathrm{OPhPy} 10$ was interesting, because this system solved the clinicity frustration by combining extension of the $\mathrm{SmA}^{*}$ temperature range with subphase generation. Figure $2 d$ shows a contact sample of this system at a temperature which is too high for the $\mathrm{SmC}^{*}$ phase to have appeared. The $\mathrm{SmC}_{\mathrm{a}}{ }^{*}, \mathrm{SmC}_{\gamma}{ }^{*}, \mathrm{SmC}_{\beta} *$ and $\mathrm{SmA}^{*}$ phases are however easy to recognize. Most likely, there is a $\mathrm{SmC}_{\alpha}{ }^{*}$ phase between $\mathrm{SmC}_{\beta} *$ and $\mathrm{SmA}^{*}$, but the homeotropic texture of this phase is identical to that of $\mathrm{SmA}^{*}$, hence it cannot be distinguished in the picture.

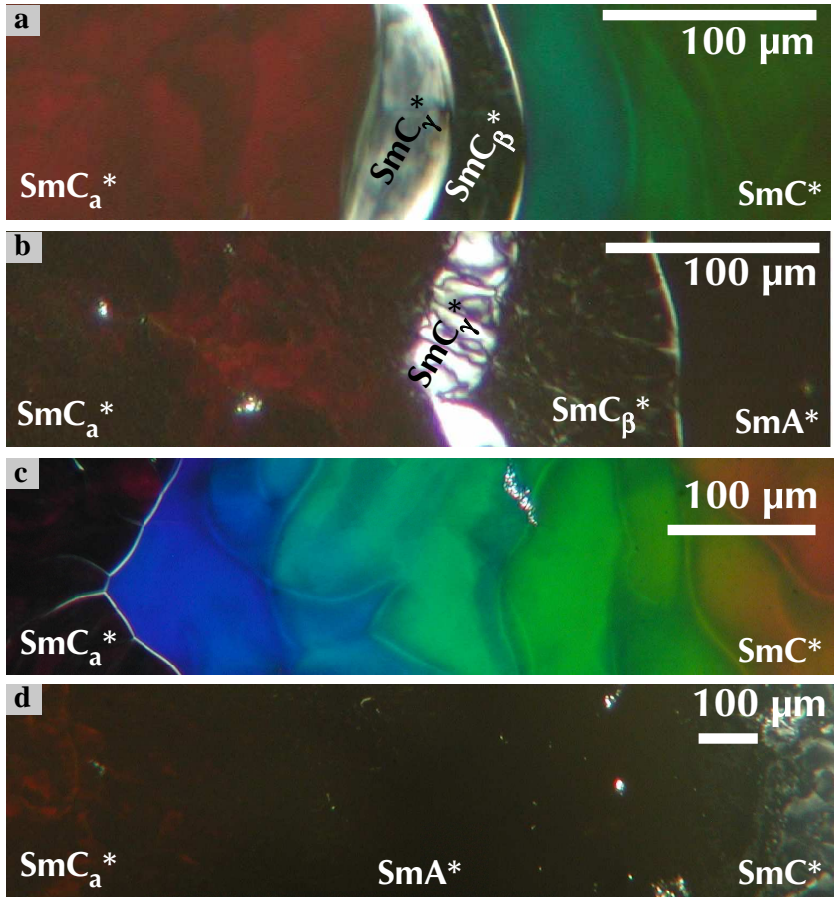

Fig. 2. Examples of the three different paths between synand anticlinic order encountered in the study, as observed with homeotropically aligned contact samples in the polarizing microscope. (a) $(S)$-TFMHPOBC (left) with $(S)$-DOBAMBC (right) at $96^{\circ} \mathrm{C}$; transition via subphases. (b) $(S, S)$-M7BBM7 (left) with $8 \mathrm{OPhPy} 10$ (right) at $92^{\circ} \mathrm{C}$; transition via subphases and extended $\mathrm{SmA}^{*}$. (c) $(S, S)$-M7BBM7 (left) with $(S)$-S1B8 (right) at $113^{\circ} \mathrm{C}$; direct $\mathrm{SmC}^{*}-\mathrm{SmCa}_{\mathrm{a}}{ }^{*}$ transition. (d) ( $S$ )TFMHPOBC (left) with $(S)-\mathrm{M} 8$ (right) at $69^{\circ} \mathrm{C}$; transition via extended $\mathrm{SmA}^{*}$.

On further cooling, the $\mathrm{SmC} / \mathrm{SmC}^{*}$ phase formed on the $8 \mathrm{OPhPy} 10$ side of the contact sample, but it never reached the subphase region; the $\mathrm{SmA}^{*}$ phase separated $\mathrm{SmC}^{*}$ from the AFLC phase sequence down to crystallization.

\subsubsection{Detailed studies on three selected systems}

We selected three mixture systems for more detailed studies, preparing homogeneous mixtures at a number of mixture ratios and carrying out texture investigations, dielectric and electrooptic studies on each mixture. In all three systems the anticlinic component was $(S)$-TFMHPOBC, but as the synclinic component was varied the path to synclinicity changed from direct $[(S)$-MHPOdCBC as synclinic], via subphases $[(S)$-DOBAMBC] to transition via extended $\mathrm{SmA}^{*}[(S)-\mathrm{S} 1 \mathrm{~B} 8]$. Together these three systems thus represent all paths found between syn- and anticlinicity. Their phase diagrams are shown in Figure 3.

In the dielectric spectrum of the 50/50 mixture of TFMHPOBC and MHPOdCBC (Figure 4a), representative of the systems with a direct clinicity change, the $\mathrm{SmC}^{*}-\mathrm{SmC}_{\mathrm{a}}$ * transition can easily be recognized. Whereas the low-temperature $\mathrm{SmC}_{\mathrm{a}}$ * regime is characterized by an almost featureless spectrum, the $\mathrm{SmC}^{*}$ phase above the 

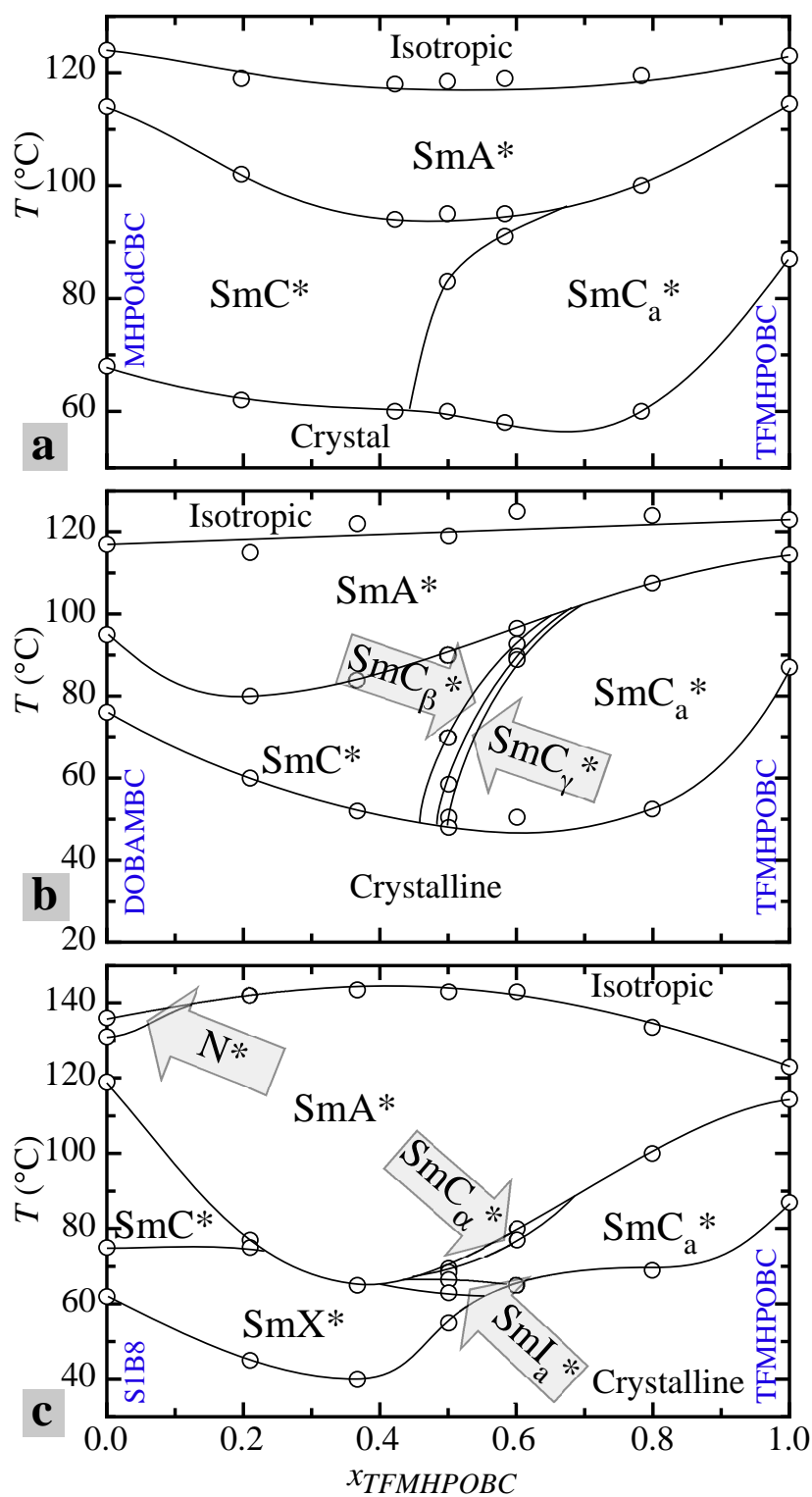

Fig. 3. Phase diagrams of three systems exhibiting the three different types of transition between syn- and anticlinic: (a) $(S)$-TFMHPOBC and $(S)$-MHPOdCBC (direct transition), (b) $(S)$-TFMHPOBC and $(S)$-DOBAMBC (transition via subphases) and (c) $(S)$-TFMHPOBC and $(S)$-S1B8 (transition via extended $\left.\mathrm{SmA}^{*}\right)$. The temperature range of untilted $\mathrm{SmA}^{*}$ is in all cases extended by mixing, hence the tendency of resolving the clinicity frustration by tilt disappearance can be spotted even in (a) and (b). Rings are actually studied transitions, lines are interpolations based on the experimental data.

phase transition exhibits a strong phason mode absorption. The only other major change in the response occurs at the $\mathrm{SmC}^{*}-\mathrm{SmA}^{*}$ transition, where the soft mode (the fluctuation corresponding to the electroclinic effect, i.e. field-induced director tilt) has its maximum and then decreases rapidly in strength on further heating.

The absorption spectrum of a 49.9/50.1 mixture of $(S)$-TFMHPOBC and $(S)$-DOBAMBC is shown in Figure $4 \mathrm{~b}$. The induced $\mathrm{SmC}_{\gamma}{ }^{*}$ and $\mathrm{SmC}_{\beta} *$ subphases are
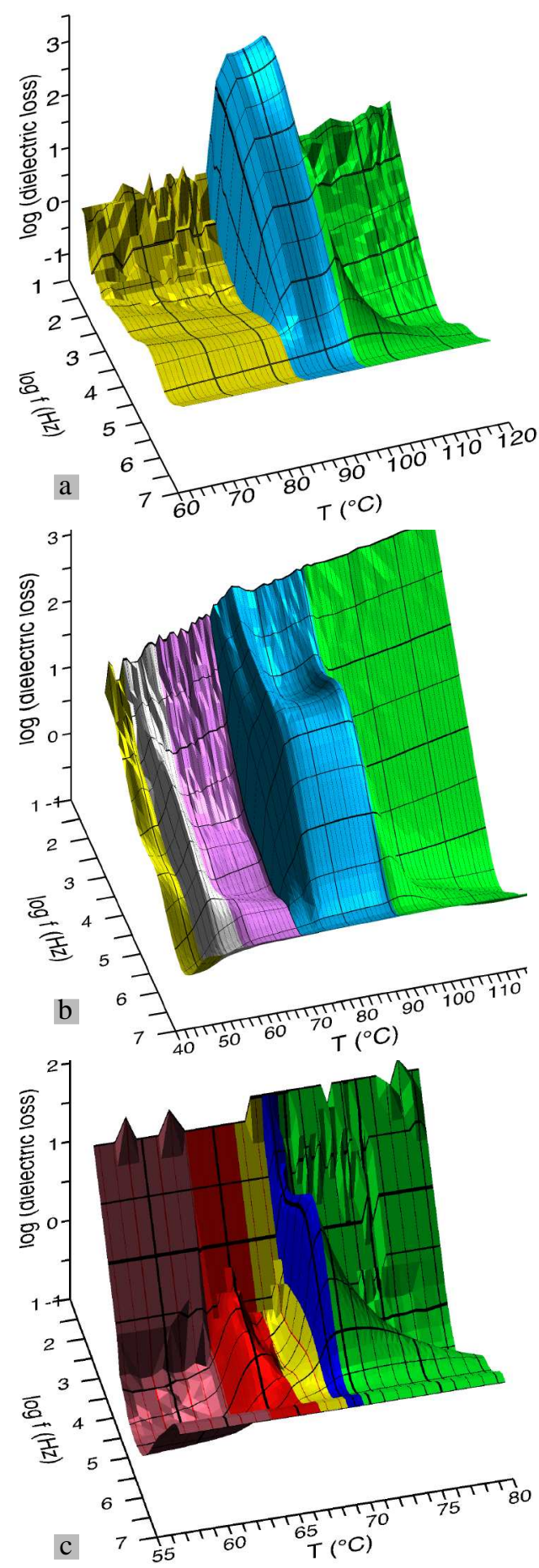

Fig. 4. Dielectric absorption spectra of (a) a 50/50 (mol $\%)$ mixture of $(S)$-TFMHPOBC and $(S)$-MHPOdCBC, (b) a $49.9 / 50.1$ (mol \%) mixture of $(S)$-TFMHPOBC and $(S)$ DOBAMBC and (c) a 53.5/46.5 (mol \%) mixture of $(S)$ TFMHPOBC and $(S)$-S1B8. All spectra were obtained on heating using $23.5 \mu \mathrm{m}$ thick planar-aligned samples. Color coding: $\mathrm{SmCa}^{*}$ - yellow, $\mathrm{SmC}_{\gamma}{ }^{*}-$ grey, $\mathrm{SmC}_{\beta}{ }^{*}$ - purple, $\mathrm{SmC}_{\alpha}{ }^{*}$ - dark blue, $\mathrm{SmC}^{*}$ - light blue, $\mathrm{SmA}^{*}$ - green, $\mathrm{SmI}^{*}{ }^{*}$ - red, SmX* - burgundy. Most likely, the (b) mixture exhibits the $\mathrm{SmC}_{\alpha}{ }^{*}$ phase in a small temperature range between $\mathrm{SmA}^{*}$ and $\mathrm{SmC}^{*}$ but due to phase coexistence phenomena this cannot clearly be separated from the surrounding phases in the dielectric spectrum. The phases below $\mathrm{SmA}^{*}$ in (c) are not yet fully confirmed. 
easy to distinguish by their characteristic responses, $\mathrm{SmC}_{\gamma}{ }^{*}$ (plotted in grey) exhibiting a clear absorption, $\mathrm{SmC}_{\beta} *$ (purple) being basically absorption-free. Surrounding these phases we can recognize the strong absorption of the $\mathrm{SmC}^{*}$ phase at higher temperatures (blue) and two weak $\mathrm{SmC}_{\mathrm{a}}{ }^{*}$ absorptions at lower temperatures (yellow). In some other systems where the subphases were induced, for instance the combinations of $(S, S)$-M7BBM7 and HOAB (see [18] where this mixture system is described in detail) or $(S)$ TFMHPOBC and $(S)$-W504, the two subphases were basically indistinguishable in the dielectric spectrum, most likely a result of phase coexistence and / or a very small value of $P_{s}$.

By careful adjustment of the mixing proportions such that the tendencies for syn- and anticlinicity are evenly balanced, the temperature range of the $\mathrm{SmC}_{\beta} *$ and / or $\mathrm{SmC}_{\gamma} *$ phase can be extended considerably. In [18] we describe the detailed study of the $(S, S)$-M7BBM7 and HOAB system within which we prepared a mixture with a $50 \mathrm{~K}$ wide $\mathrm{SmC}_{\beta} *$ phase. Within the $(S)$-TFMHPOBC and $(S)$-DOBAMBC system, we prepared only five mixtures, giving poor resolution in the interesting range around 50/50 composition. The phase transition curves for this system in Figure $3 \mathrm{c}$ are estimates based on the observations made on the five mixtures prepared. It is likely that the real curves relating to the subphase transitions are closer to vertical close to the balanced mixture ratio, i.e. we can expect larger temperature ranges of $\mathrm{SmC}_{\beta}$ * and / or $\mathrm{SmC}_{\gamma} *$ than what we observed with the mixture studied in Figure $4 \mathrm{~b}$.

The system chosen to represent the transition via extended $\mathrm{SmA}^{*}$ phase, TFMHPOBC $+\mathrm{S} 1 \mathrm{~B} 8$, turned out to be more complex than the initial contact sample texture studies had suggested. Careful investigations of mixtures in the vicinity of 50/50 composition revealed that the texture at low temperatures was not completely black, but exhibited very weak dark grey schlieren. Dielectric spectroscopy measurements on each prepared mixture (five in total, cf. the phase diagrams in Figure 3) also clearly showed that the $\mathrm{SmA}^{*}$ phase never extended lower than about $65^{\circ} \mathrm{C}$ in any mixture. In the mixtures with $20.7 \%$ and $53.5 \%$ TFMHPOBC, the temperature range below $\mathrm{SmA}^{*}$ was dominated by a phase that exhibited no response to electric fields (no electrooptic switching and almost no dielectric absorption), but which clearly is liquid crystalline as it was mechanically shearable. Its viscosity was however considerably higher than in $\mathrm{SmA}^{*}$, so we assume this to be a higher-ordered smectic phase, for now simply denoted $\mathrm{SmX}^{*}$ (also in the investigations of pure S1B8 of Schacht et al. [30] two higher-order smectic phases were detected, but not identified). The mixture with $53.5 \%$ TFMHPOBC exhibited the most complex phase sequence within this system, with the $\mathrm{SmA}^{*}$ phase on cooling being followed first by a thin $\mathrm{SmC}_{\alpha}{ }^{*}$ phase, then $\mathrm{SmC}_{\mathrm{a}}{ }^{*}$, and thereafter a phase which we believe is $\mathrm{SmI}_{\mathrm{a}}{ }^{*}$, before entering the unidentified $\mathrm{SmX}^{*}$ phase, cf Figure 4c. The $\mathrm{SmC}_{\alpha}{ }^{*}$ phase may be difficult to recognize in this figure, but it was easily detectable in the mixture with $61.3 \%$ TFMHPOBC (not shown), where its temper-

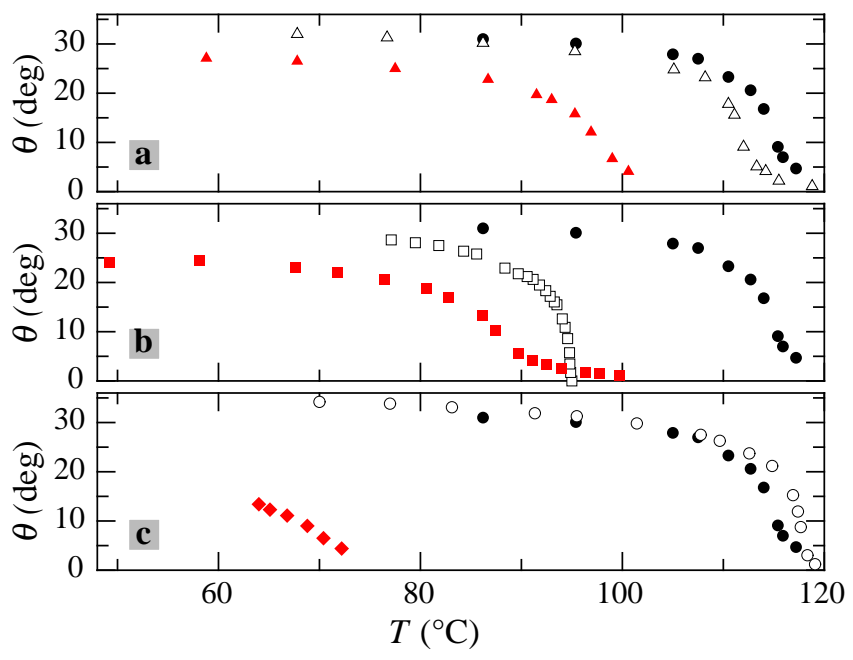

Fig. 5. The optical tilt angle of the four components used for the three mixtures systems selected for detailed studies, and for $\sim 50 / 50$ mixtures in each system. The anticlinic is in all cases TFMHPOBC (filled black circles) and the synclinics are (a) MHPOdCBC (empty triangles), (b) DOBAMBC (empty squares, data from Dumrongrattana et al. [31]) and (c) S1B8 (empty circles, data from Schacht et al. [30]). The respective mixture data are plotted with red symbols.

ature range was somewhat larger. A weak polar response together with a perfectly black homeotropic texture, indistinguishable from $\mathrm{SmA}^{*}$, serve as the basis for our designating the phase as $\mathrm{SmC}_{\alpha} *$.

Since the $\mathrm{SmX}^{*}$ phase may be tilted it is possible that the director tilt does not disappear completely in this mixture system. However, in the phase diagram the $\mathrm{SmC}^{*}$ and $\mathrm{SmC}_{\mathrm{a}} *$ phase are horizontally fully separated by $\mathrm{SmA}^{*}$ (cf. Figure 3), thereby resolving the clinicity frustration. Even if $\mathrm{SmX}^{*}$ is tilted, the low-temperature end of $\mathrm{SmA}^{*}$ was in the $41.2 \%$ mixture detected at about $65^{\circ} \mathrm{C}, 50$ $55 \mathrm{~K}$ lower than the temperature of onset of tilt in the respective pure components and even below the melting point of $(S)$-TFMHPOBC, so the suppression of tilt is quite impressive. As expected, it is this system which exhibits the smallest tilt values in Figure 5. When the mixture entered the $\mathrm{SmI}_{\mathrm{a}}$ * phase the measured tilt angle value decreased compared to that measured at higher temperatures. Most likely this is an artifact due to the too high switching threshold, hence these data are not shown in the figure. A certain general reduction in tilt angle is actually seen in both other mixture systems as well, which correlates well with the fact that the $\mathrm{SmA}^{*}$ phase has its maximum temperature range at intermediate mixing ratios for all systems studied.

\subsubsection{Relation between transition path and choice of mixture components}

An overview of how the path depended on the compound combination is given in Table 3 . We first note that DOBAMBC and IPC-16 induce the intermediate subphases when mixed 
Table 3. The paths between syn- and anticlinic order as each anticlinic is mixed with different synclinic-forming compounds.

\begin{tabular}{|c|c|c|c|c|c|}
\hline & EHPOCBC & EHPDoCBC & TFMHPOBC & TFMHPBC-11 & M7BBM7 \\
\hline DOBAMBC & 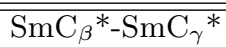 & 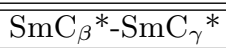 & 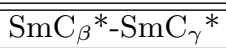 & 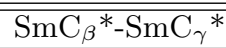 & 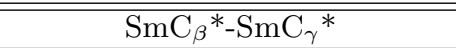 \\
\hline A8 & $\mathrm{SmA}^{*}$ & $\mathrm{SmA}^{*}$ & $\mathrm{SmA}^{*}$ & $\mathrm{SmA}^{*}$ & $\mathrm{SmA}^{*}$ \\
\hline M8 & $\mathrm{SmA}^{*}$ & direct & $\mathrm{SmA}^{*}$ & $\mathrm{SmA}^{*}$ & direct \\
\hline S1B8 & $\mathrm{SmA}^{*}$ & $\mathrm{SmA}^{*}$ & $\mathrm{SmA}^{*}$ & $\mathrm{SmA}^{*}$ & direct \\
\hline IPC16 & $\mathrm{SmC}_{\beta}{ }_{-}-\mathrm{SmC}_{\gamma}{ }^{*}$ & $\mathrm{SmC}_{\beta}{ }^{*} \mathrm{SmC}_{\gamma}{ }^{*}$ & $\mathrm{SmC}_{\beta}{ }^{*}-\mathrm{SmC}_{\gamma} *$ & $\mathrm{SmC}_{\beta}{ }_{-}-\mathrm{SmC}_{\gamma}{ }^{*}$ & $\mathrm{SmC}_{\beta}{ }^{*} \mathrm{SmC}_{\gamma}{ }^{*}$ \\
\hline MHPOdCBC & $\mathrm{SmC}_{\beta}{ }^{*}-\mathrm{SmC}_{\gamma}{ }^{*}$ & $\mathrm{SmC}_{\beta}{ }^{*} \mathrm{SmC}_{\gamma}{ }^{*}$ & direct & $\mathrm{SmC}_{\beta}{ }^{*}-\mathrm{SmC}_{\gamma}{ }^{*}$ & $\mathrm{SmC}_{\beta}{ }^{*}-\mathrm{SmC}_{\gamma}{ }^{*}$ \\
\hline W504 & $\mathrm{SmC}_{\beta}{ }_{-} \mathrm{SmC}_{\gamma}{ }^{*}$ & direct & $\mathrm{SmC}_{\beta}{ }_{-} \mathrm{SmC}_{\gamma}{ }^{*}$ & $\mathrm{SmC}_{\beta}{ }^{*}-\mathrm{SmC}_{\gamma}{ }^{*}$ & direct \\
\hline 8OPhPy10 & $\mathrm{SmA}^{*}$ & $\mathrm{SmA}^{*}$ & $\mathrm{SmA}^{*}$ & $\mathrm{SmA}^{*}$ & $\mathrm{SmA}^{*}, \mathrm{SmC}_{\alpha}{ }^{*}, \mathrm{SmC}_{\beta}{ }^{*}, \mathrm{SmC}_{\gamma}{ }^{*}$ \\
\hline HOAB & $\mathrm{SmA}^{*}$ & $\mathrm{SmA}^{*}$ & $\mathrm{SmA}^{*}$ & $\mathrm{SmA}^{*}$ & $\mathrm{SmC}_{\beta}{ }_{-}-\mathrm{SmC}_{\gamma}{ }^{*}$ \\
\hline
\end{tabular}

with any anticlinic mesogen used in the study. The success of the former compound in generating the subphases was quite a surprise, considering the proposal that the subphases require strong chiral interactions. DOBAMBC has a very long $\mathrm{SmC}^{*}$ pitch and a low saturation value of $P_{s}$, hence it would seem to be a clear case of weak chiral interactions. Subphases are also the most common route when the synclinic compound is MHPOdCBC or W504, although the direct transition is also observed in these cases. All these compounds exhibit a second-order SmA*$\mathrm{SmC}^{*}$ transition, whereas A8, M8 and S1B8, which never produce the $\mathrm{SmC}_{\beta}{ }^{*}$ and $\mathrm{SmC}_{\gamma}{ }^{*}$ phases, have first-order $\mathrm{SmA}^{*}-\mathrm{SmC}^{*}$ or $\mathrm{N}^{*}-\mathrm{SmC}^{*}$ (M8) transitions. The achiral synclinics, both of which produce subphases when mixed with $(S, S)$-M7BBM7, have second-order SmA-C transition and first-order N-C transition, respectively. The observations thus give a hint that the order of the tilting transition may be important for the path between synand anticlinicity which is followed, such that a first-order $\mathrm{SmA}^{*}{ }_{-} \mathrm{SmC}^{*}$ transition is incompatible with the generation of the intermediate phases. However, we have in this investigation had access to only two synclinics with firstorder $\mathrm{SmA}^{*}{ }_{-} \mathrm{SmC}^{*}$ transition, having certain similarities in the molecule constitution, hence there might be other reasons why these mesogens do not allow the route via subphases. This possible trend must thus be corroborated with further investigations, using first-order $\mathrm{SmA}^{*}-\mathrm{SmC}^{*}$ synclinics with substantially different molecule structures.

IPC-16 and MHPOdCBC both have very long achiral chains rendering these molecules considerably longer than any anticlinic with which they are mixed. In a study of how molecules of different lengths mix in smectic phases, Keymeulen and co-workers concluded that the longer of the mesogens may adopt a strongly kinked conformation of one of its end chains, thereby shortening the effective molecule length such that packing with the shorter molecules can still be efficient [32]. It has been suggested that one of the most important structural features for promoting an AFLC phase sequence is a end chain which prefers to be non-parallel to the core, angles in the range of $90^{\circ}$ being typical for the prototype chiral AFLC tail, 1-methylheptyloxycarbonyl [33-35]. This geometrical feature prevents out-of-layer fluctuations and thus increases the degree of smectic order. We may spectulate that the long achiral tails of IPC-16 and of MHPOdCBC are not extended and straight in our mixtures but kinked into
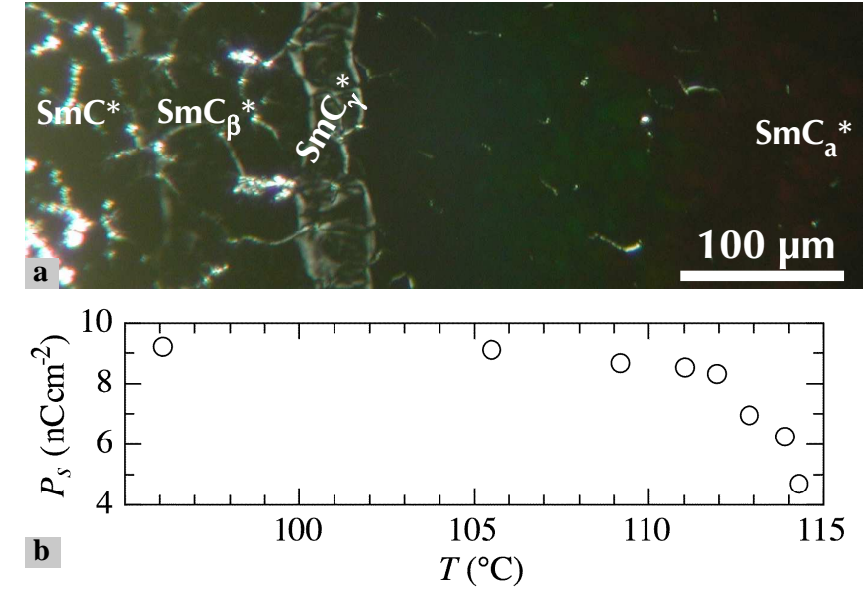

Fig. 6. The combination of TFMHPOBC and W504 investigated in a contact sample (a) and in a 51/49 mixture, measuring the spontaneous polarization (b).

a hook shape, thereby increasing the smectic order and hence facilitating subphase formation. Looking at the role of the anticlinic, finally, it is much more difficult to spot any pattern regarding the clinicity change route. Each anticlinic can follow the subphase route as well as the route via extended $\mathrm{SmA}^{*}$ and three of the five also show the direct transition.

Based on the observation that AFLCs generally have high spontaneous polarization, one might think that the magnitude of $P_{s}$ plays a role for the route which is followed. The anticlinics in the study have saturation values of $P_{s}$ between 42 and $95 \mathrm{nCcm}^{-2}$, and many of the FLCs had much higher magnitude of polarization. There was no correlation between the magnitude of $P_{s}$ of the pure components and the type of transition between synand anticlinic order which resulted. Moreover, as most of the AFLCs had $\mathbf{P}_{s}$ of different sign from that of the FLCs with which they were mixed the mixtures generally passed through a point of zero spontaneous polarization, but there was no clear consequences of this on the phase sequences observed. For instance, a mixture of $(S)$-TFMHPOBC and $(S)$-W504 in 51/49 molar ratio exhibited a $\mathrm{SmC}^{*}-\mathrm{SmC}_{\beta}{ }^{*}-\mathrm{SmC}_{\gamma} *$ phase sequence but the spontaneous polarization did not even reach $10 \mathrm{nCcm}^{-2}$, cf. Figure 6, a very low value indeed for an AFLC. 
The fact that the $\mathrm{SmC}_{\beta} *$ and $\mathrm{SmC}_{\gamma} *$ subphases were frequently generated in our mixtures of syn- and anticlinics lends strong support to the initial notion that the main prerequisite for the formation of these phases is the frustration between syn- and anticlinicity and / or between syn- and antipolarity. Akizuki et al. have shown that director configurations which are qualitatively identical to those shown for these two phases in Figure 1 can very simply be generated by combining syn- and anticlinic layer interfaces [11]. If we to the synclinic structure of $\mathrm{SmC}^{*}$ add a competing tendency for anticlinic order by setting every second layer interface anticlinic, we get the four-layer period director configuration of $\mathrm{SmC}_{\beta}{ }^{*}$, the first phase to follow $\mathrm{SmC}^{*}$ on cooling. In doing so, one must remember that the small chiral modulation between every two layers that corresponds to the helical superstructure, and which thus comes on top of the basic $0^{\circ}$ or $180^{\circ}$ change in tilting direction, is in opposite directions for syn- and anticlinic layer interfaces (the helical modulation must change sense at a transition between syn- and anticlinic [36]). If we assume the magnitude of the chiral modulation to be constant the resulting phase gets an infinite pitch. Indeed, the $\mathrm{SmC}_{\beta}$ * phase is known to have very long pitch and a helix inversion is often encountered within or at a border of this phase, see e.g. [37]. The cases where its pitch is long but not infinite can easily be accounted for by assuming slightly different magnitudes of the chiral modulation at syn- and anticlinic interfaces. To arrive at the structure of $\mathrm{SmC}_{\gamma}{ }^{*}$ the same scheme is applied but now with two anticlinic interfaces between every synclinic, reflecting the fact that this phase is closer to the fully anticlinic $\mathrm{SmC}_{\mathrm{a}}{ }^{*}$ phase.

The difference between the results of this type of construction and the experimentally determined structures is the angle between directors at synclinic interfaces, which in experimental work has been found to be on the order of $20^{\circ}$ for $\mathrm{SmC}_{\beta} *$ and even higher for $\mathrm{SmC}_{\gamma} *[13,15]$, whereas it with the Akizuki method becomes the same as in $\mathrm{SmC}^{*}$, typically $2-3^{\circ}$. This difference shows that the method of combining syn- and anticlinic interfaces, each assumed to be unaffected by the presence of neighbor interfaces of opposite type, is too simple. Nevertheless, the basic approach seems sound and is clearly supported by the present experimental data.

\section{Conclusions and outlook}

In our study of induced frustration between the synclinic $\mathrm{SmC}^{*}$ and anticlinic $\mathrm{SmC}_{\mathrm{a}}$ * phases we could identify three possible paths between the two incompatible types of order:

- transition via the subphases $\mathrm{SmC}_{\beta} *$ and $\mathrm{SmC}_{\gamma}{ }^{*}$

- transition via tilt disappearance, i.e. via extended SmA* phase

- direct transition $\mathrm{SmC}^{*}-\mathrm{SmC}_{\mathrm{a}}{ }^{*}$.

The most common was the first one, demonstrating that the fundamental requirement for the appearance of the $\mathrm{SmC}_{\beta}$ * and $\mathrm{SmC}_{\gamma}{ }^{*}$ phases is the frustration between syn- and anticlinicity. By deliberately inducing such frustration, like in the mixtures studied here, one can open completely new possibilities to study theses phases in detail. First of all, the temperature range can be widely extended compared to the typical $0.5-5 \mathrm{~K}$ ranges in pure substances. Second, it may be very interesting to apply the revealing resonant $\mathrm{x}$-ray methodology $[14,15]$ to mixtures of this type. By mixing a synclinic compound containing a sulphur atom in the core with a selenium-containing anticlinic, or vice versa, it would be possible to study each component separately by tuning the x-ray frequency. In this way one can investigate if the frustration also leads to some microphase segregation between components preferring synclinic order and those striving for anticlinic organization, or if the phase is fully homogeneous.

Even with non-chiral synclinic compounds the path via induced subphases was observed, and the low-polarization and long-pitch FLC $(S)$-DOBAMBC was one of the most successful synclinics in generating the subphases. Furthermore, the subphase path was also followed in mixture systems where the spontaneous polarization was reduced to very low magnitude. Although chirality and spontaneous polarization are inherent qualities of all AFLCs, materials displaying a phase sequence comprising the subphases are thus not exceptional in these respects. A relevant followup study for understanding the role of chirality better, will be to combine $(S)$ - and $(R)$-enantiomers, one being synclinic the other anticlinic.

The $\mathrm{SmC}_{\alpha}{ }^{*}$ subphase was detected over a small mixture ratio range in a system that took the syn- to anticlinicity path via extended $\mathrm{SmA}^{*}$, but where no $\mathrm{SmC}_{\beta}{ }^{*}$ or $\mathrm{SmC}_{\gamma}{ }^{*}$ phase was detected. Whereas the two latter generally appear together, obviously as intermediate states between syn- and anticlinicity, the former seems more related to the $\mathrm{SmC}^{*}$ phase. In our set of nine synclinics and five anticlinics the path chosen between syn- and anticlinicity was determined mainly by the synclinic component: if this had a first-order tilting transition subphases were never induced. In contrast, with a second-order Sm$A^{*}-\mathrm{Sm}-C^{*}$ transition the subphases were almost always induced, a direct transition being the exception in three cases.

Financial support from the Alexander von Humboldt foundation (JPFL) is gratefully acknowledged. We are indebted to E. Körblova (University of Colorado) for providing the sample of W504.

\section{References}

1. S. T. Lagerwall, Ferroelectric and Antiferroelectric Liquid Crystals. Weinheim: Wiley-VCH, 1999.

2. J. P. F. Lagerwall, F. Giesselmann, A. Saipa, and R. Dabrowski, "On the origin of high optical director tilt in a partially fluorinated orthoconic antiferroelectric liquid crystal," Liq. Cryst., vol. 31, no. 9, pp. 1175-1184, 2004. 
3. M. Hamaneh and P. Taylor, "Long-range interlayer interactions in ferroelectric liquid crystals," Phys. Rev. Lett., vol. 93, no. 16, p. 167801, 2004.

4. A. Emelyanenko and M. Osipov, "Theoretical model for the discrete flexoelectric effect and a description for the sequence of intermediate smectic phases with increasing periodicity," Phys. Rev. E, vol. 68, no. 5, p. 051703, 2003.

5. M. Cepic and B. Zeks, "Flexoelectricity and piezoelectricity: the reason for the rich variety of phases in antiferroelectric smectic liquid crystals," Phys. Rev. Lett., vol. 87, no. 8, p. 085501, 2001.

6. A. Chandani, Y. Ouchi, H. Takezoe, and A. Fukuda, "Novel phases exhibiting tristable switching," Jap. J. Appl. Phys. 2, vol. 28, no. 7, pp. L1261-4, 1989.

7. J. P. F. Lagerwall, P. Rudquist, S. T. Lagerwall, and F. Giesselmann, "On the phase sequence of antiferroelectric liquid crystals and its relation to orientational and translational order," Liq. Cryst., vol. 30, no. 4, pp. $399-$ 414, 2003.

8. R. Dabrowski, "Liquid crystals with fluorinated terminal chains and antiferroelectric properties," Ferroelectrics, vol. 243 , no. $1-4$, pp. 1-18, 2000.

9. J. Gasowska, R. Dabrowski, W. Drzewinski, M. Filipowicz, J. Przedmojski, and K. Kenig, "Comparison of mesomorphic properties in chiral and achiral homologous series of high tilted ferroelectrics and antiferroelectrics," Ferroelectrics, vol. 309, pp. 83-93, 2004.

10. T. Isozaki, T. Fujikawa, H. Takezoe, A. Fukuda, T. Hagiwara, Y. Suzuki, and I. Kawamura, "Competition between ferroelectric and antiferroelectric interactions stabilizing varieties of phases in binary mixtures of smectic liquid crystals," Jap. J. Appl. Phys. 2, vol. 31, no. 10A, pp. L1435-8, 1992.

11. T. Akizuki, K. Miyachi, Y. Takanishi, K. Ishikawa, H. Takezoe, and A. Fukuda, "Molecular orientational structures with macroscopic helix in antiferroelectric liquid crystal subphases," Jap. J. Appl. Phys. 1, vol. 38, no. 8, pp. 4832-4837, 1999.

12. M. Osipov, A. Fukuda, and H. Hakoi, "Synclinic and anticlinic ordering in frustrated smectics," Mol. Cryst. Liq. Cryst., vol. 402, pp. 245-266, 2003.

13. P. M. Johnson, D. A. Olson, S. Pankratz, H. T. Nguyen, J. W. Goodby, M. Hird, and C. C. Huang, "Structure of the liquid-crystal ferrielectric phases as determined by ellipsometry," Phys. Rev. Lett., vol. 84, no. 21, pp. 48704873, 2000.

14. P. Mach, R. Pindak, A. M. Levelut, P. Barois, H. T. Nguyen, C. C. Huang, and L. Furenlid, "Structural characterization of various chiral smectic-c phases by resonant x-ray scattering," Phys. Rev. Lett., vol. 81, no. 5, pp. 10151018, 1998.

15. A. Cady, J. A. Pitney, R. Pindak, L. S. Matkin, S. J. Watson, H. F. Gleeson, P. Cluzeau, P. Barois, A. M. Levelut, W. Caliebe, J. Goodby, M. Hird, and C. C. Huang, "Orientational ordering in the chiral smcfi2* liquid crystal phase determined by resonant polarized x-ray diffraction," Phys. Rev. E, vol. 64, p. 050702, 2001.

16. E. Gorecka, D. Pociecha, M. Cepic, B. Zeks, and R. Dabrowski, "Enantiomeric excess dependence of the phase diagram of antiferroelectric liquid crystals," Phys. Rev. E, vol. 65, p. 061703, 2002.

17. M. Cepic, E. Gorecka, D. Pociecha, B. Zeks, and H. Nguyen, "Theoretical and experimental study of the intermediate smcfi2 $\left(^{*}\right)$ and the $\operatorname{smcfi1}(*)$ phases in antiferroelectric liquid crystals," J. Chem. Phys., vol. 117, no. 4, pp. $1817-1826,2002$.

18. J. P. F. Lagerwall, F. Giesselmann, C. Selbmann, S. Rauch, and G. Heppke, "Generation of frustrated liquid crystal phases by mixing an achiral nsmc mesogen with an antiferroelectric chiral smectic liquid crystal," J. Chem. Phys., vol. 122, no. 14, p. 144906, 2005.

19. T. Isozaki, T. Fujikawa, H. Takezoe, A. Fukuda, T. Hagiwara, Y. Suzuki, and I. Kawamura, "Devil's staircase formed by competing interactions stabilizing the ferroelectric smectic-c* phase and the antiferroelectric smectic$\mathrm{c}^{*} /$ sub a/ phase in liquid crystalline binary mixtures," Phys. Rev. B, vol. 48, no. 18, pp. 13439-13450, 1993.

20. A. Fukuda, Y. Takanishi, T. Isozaki, K. Ishikawa, and H. Takezoe, "Antiferroelectric chiral smectic liquid crystals (feature article)," J. Mater. Chem., vol. 4, no. 7, pp. 9971016, 1994.

21. S.-S. Seomun, T. Gouda, Y. Takanishi, K. Ishikawa, and H. Takezoe, "Bulk optical properties in binary mixtures of antiferroelectric liquid crystal compounds showing vshaped switching," Liq. Cryst., vol. 26, no. 2, pp. 151 $161,1999$.

22. E. Gorecka, D. Pociecha, M. Glogarova, and J. Mieczkowski, "Observation of a frustrated phase in mixtures of ferroelectric and antiferroelectric liquid crystals," Phys. Rev. Lett., vol. 81, no. 14, pp. 2946-2949, 1998.

23. D. Pociecha, M. Glogarova, E. Gorecka, and J. Mieczkowski, "Behavior of frustrated phase in ferroelectric and antiferroelectric liquid crystalline mixtures," Phys. Rev. E, vol. 61, no. 6, pp. 6674-6677, 2000.

24. F. Giesselmann, A. Langhoff, and P. Zugenmaier, "Dispersion of the optical axes in smectic c* liquid crystals," Liq. Cryst., vol. 23, no. 6, pp. 927-931, 1997.

25. K. Miyasato, S. Abe, H. Takezoe, and A. Fukuda, "Direct method with triangular waves for measuring spontaneous polarization in ferroelectric liquid crystals," Jap. J. Appl. Phys. 2, vol. 22, p. L661, 1983.

26. J. P. F. Lagerwall, F. Giesselmann, E. Körblova, D. M. Walba, J. M. Oton, D. Coleman, R. Shao, and N. A. Clark, "The peculiar optic, dielectric and x-ray diffraction properties of a fluorinated de vries asymmetric-diffusecone-model ferroelectric liquid crystal," submitted to Liquid Crystals, 2005.

27. A. de Vries, "The description of the smectic a and c phases and the smectic a-c phase transition of tcoob with a diffusecone model," J. Chem. Phys., vol. 71, no. 1, pp. 25-31, 1979.

28. A. de Vries, "The implications of the diffuse-cone model for smectic a and c phases and a-c phase transitions," Mol. Cryst. Liq. Cryst. Lett., vol. 49, no. 6, pp. 179-185, 1979.

29. A. de Vries, A. Ekachai, and N. Spielberg, "Why the molecules are tilted in all smectic a phases, and how the layer thickness can be used to measure orientational disorder," Mol. Cryst. Liq. Cryst. Lett., vol. 49, no. 5, pp. 143$152,1979$.

30. J. Schacht, H. Baethge, F. Giesselmann, and P. Zugenmaier, "Tilt-polarization coupling in a homologous series of ferroelectric liquid crystals," J. Mater. Chem., vol. 8, no. 3, pp. 603-612, 1998.

31. S. Dumrongrattana, C. C. Huang, G. Nounesis, S. C. Lien, and J. M. Viner, "Tilt-angle, polarization, and heat- 
capacity measurements near the smectic-a chiral-smecticc phase-transition of para-(normal-decyloxybenzylidene)para-amino-(2-methylbutyl)cinnamate (dobambc)," Phys. Rev. A, vol. 34, no. 6, pp. 5010-5019, 1986.

32. H. Keymeulen, W. de, Jeu, J. Slattery, and M. Veum, "How do smectic liquid crystals of different molecular length mix in thin films?," Eur. Phys. J. E, vol. 9, no. 5, pp. 443-446, 2002.

33. T. Nakai, S. Miyajima, Y. Takanishi, S. Yoshida, and A. Fukuda, "High-resolution c-13 nmr study of an antiferroelectric liquid crystal: Verification of the bent chain structure," J. Phys. Chem. B, vol. 103, no. 3, pp. 406-416, 1999.

34. T. Matsumoto, A. Fukuda, M. Johno, Y. Motoyama, T. Yui, S. S. Seomun, and M. Yamashita, "A novel property caused by frustration between ferroelectricity and antiferroelectricity and its application to liquid crystal displays - frustoelectricity and v-shaped switching," J. Mater. Chem., vol. 9, no. 9, pp. 2051-2080, 1999.

35. D. M. Walba, "Ferroelectric liquid crystal conglomerates," in Topics in Stereochemistry, Materials-Chirality, vol. 24 (M. M. Green, R. J. M. Nolte, E. W. Meijer, and S. E. Denmark, eds.), pp. 457-518, Wiley-VCH, 2003.

36. J. P. F. Lagerwall, F. Giesselmann, and M. A. Osipov, "On the change in helix handedness at transitions between the sm-c* and sm-ca* phases in chiral smectic liquid crystals," Submitted to Liquid Crystals, 2005.

37. I. Musevic, M. Skarabot, G. Heppke, and H. T. Nguyen, "Temperature dependence of the helical period in the ferrielectric smectic phases of mhpobc and 10otbbb1m7," Liq. Cryst., vol. 29, no. 12, pp. 1565 - 1568, 2002. 